\title{
A Perspective on Withania somnifera Modulating Antitumor Immunity in Targeting Prostate Cancer
}

\author{
Seema Dubey, ${ }^{1}$ Manohar Singh, ${ }^{1}$ Ariel Nelson, ${ }^{2}$ and Dev Karan ${ }^{1}{ }^{1}$ \\ ${ }^{1}$ Department of Pathology, MCW Cancer Center and Prostate Cancer Center of Excellence, Medical College of Wisconsin, \\ 8701 Watertown Plank Road, Milwaukee, WI 53226, USA \\ ${ }^{2}$ Department of Medicine, Division of Hematology and Oncology, Medical College of Wisconsin, 8701 Watertown Plank Road, \\ Milwaukee, WI 53226, USA
}

Correspondence should be addressed to Dev Karan; dkaran@mcw.edu

Received 2 July 2021; Accepted 7 August 2021; Published 26 August 2021

Academic Editor: Tomasz Baczek

Copyright (C) 2021 Seema Dubey et al. This is an open access article distributed under the Creative Commons Attribution License, which permits unrestricted use, distribution, and reproduction in any medium, provided the original work is properly cited.

\begin{abstract}
Medicinal plants serve as a lead source of bioactive compounds and have been an integral part of day-to-day life in treating various disease conditions since ancient times. Withaferin A (WFA), a bioactive ingredient of Withania somnifera, has been used for health and medicinal purposes for its adaptogenic, anti-inflammatory, and anticancer properties long before the published literature came into existence. Nearly $25 \%$ of pharmaceutical drugs are derived from medicinal plants, classified as dietary supplements. The bioactive compounds in these supplements may serve as chemotherapeutic substances competent to inhibit or reverse the process of carcinogenesis. The role of WFA is appreciated to polarize tumor-suppressive Th1-type immune response inducing natural killer cell activity and may provide an opportunity to manipulate the tumor microenvironment at an early stage to inhibit tumor progression. This article signifies the cumulative information about the role of WFA in modulating antitumor immunity and its potential in targeting prostate cancer.
\end{abstract}

\section{Introduction}

Since ancient times, people have used herbal medicines from plants or their extracts derived from flowers, seeds, bark, leaves, or roots to prevent or treat multiple disease types. Despite significant progress in early detection, advancement in understanding the molecular targets, and improved anticancer therapy, prostate cancer remains the second most common male malignancy and the fifth leading cause of cancer deaths among men worldwide [1]. Many useful bioactive compounds, currently in use as chemotherapy drugs, including taxanes, the camptothecin derivatives, the epipodophyllotoxins, and the vinca alkaloids, have been extracted and isolated from plant sources [2]. In addition, epidemiological studies support that dietary modification may substantially reduce a man's risk of developing prostate cancer [3, 4]. Therefore, understanding the role of plant-based therapies in determining the immune mechanisms of prostate cancer prevention and the therapeutic efficacy is crucial.

Withania somnifera, also known as Indian ginseng, is a traditional Ayurvedic medicinal plant that contains diverse bioactive compounds, including alkaloids, withanolides, and saponins. The main phytochemicals are withanolides, which are structurally similar to the ginsenosides of Panax ginseng (Asian ginseng), Panax notoginseng (Sanchi ginseng), and Panax quinquefolius (American ginseng). However, the ginsenoside contents within the Panax species vary significantly [5]. The pharmacological activity of W. somnifera is assigned to its main withanolide-Withaferin A (WFA) [6]. Although various parts of this plant are used for multiple disease treatments, root extract is a rich source of WFA. Growing evidence has shown that WFA has anti-inflammatory, antimicrobial, and anticancer activities [7-10]. This review highlights the effect of WFA in regulating both the 
immunological and nonimmunological targets and its potential as an anticancer agent for prostate carcinoma.

\section{Nonimmune Molecular Targets of WFA}

Withaferin A is a nontoxic, bioactive compound of Withania somnifera, a widely used traditional medicine in Asia and Africa for its anticancer activities and to enhance the immunological response. Numerous studies have described the anticancer effect of WFA in various cancer types, including leukemia, melanoma, prostate cancer, breast cancer, ovarian cancer, head and neck cancer, and colon cancer [11-14]. WFA inhibits cell proliferation, invasion, metastasis, angiogenesis, proteasome, endoplasmic reticulum (ER) stress, protein folding, and maturation in cancer cells and regulates multiple targets by direct interaction or regulation of secondary targets in establishing its anticancer activity. Since several succinct review articles have described the role of WFA in various cancer types $[11,12,15]$, we limit our discussion to prostate cancer along with important molecular targets from other cancer types.

2.1. WFA-Mediated Cell Cycle Inhibition. Analysis of cell cycle-related events is a primary mechanism to examine a natural compound's biological effect in targeting cancer cells. WFA inhibits cell proliferation by inducing cell cycle arrest in the G2/M phase in multiple studies [16-20]. Dysregulation in the cell cycle process is associated with prostate cancer development, and currently, several cell cycle inhibitors are being evaluated in clinical trials for prostate cancer treatment [21]. In prostate cancer cell lines PC3 and DU145, WFA showed cell cycle inhibition in the G2/M phase by upregulating p21, phosphorylated wee-1, phosphorylated histone $H 3$, and aurora B and downregulating cyclin (A2, B1, and E2) expression [22]. Interestingly, WFA showed a higher cytotoxic effect in androgen-resistant, androgen receptor (AR) negative cell lines PC3 and DU145 than androgen-sensitive, AR positive LNCaP cells [23]. In addition, WFA increased prostate apoptosis response- 4 protein (Par-4) to enhance proapoptotic signaling in prostate cancer cells [14].

Following other targeted mechanisms, WFA decreased cyclin-dependent kinase (Cdk1), cell division cycle (Cdc 25B), and Cdc 25C expression and arresting cell cycle in the $\mathrm{G} 2 / \mathrm{M}$ phase in breast cancer [18]. WFA prevents Cdk1/cyclin B1 complex formation, a critical step of cell cycle progression in gastric cancer, by dephosphorylating Cdk1 at Thr161 and p21 upregulation in glioblastome [16]. Additional studies showed that increased oxidative stress is critical in the WFA-mediated cell cycle and cell proliferation inhibition [17]. WFA-directed reticence in cell proliferation and migration is credited to blocking STAT3 transcription activity in colon cancer [24]. More importantly, combined treatment of WFA and liposomal preparation of doxorubicin enhanced cell death in ovarian cancer cells and inhibited the expression of cancer stem cell markers ALDH1 (aldehyde dehydrogenase) and Notch1 [25]. WFA is also known to restore tumor necrosis factor-related apoptosis-inducing ligand (TRAIL) sensitivity inducing apoptosis in human renal and breast cancer cells $[26,27]$.
2.2. Proteasomal Inhibition by WFA. Ubiquitin proteasome plays a critical role in neoplastic cell growth, survival, and apoptosis by reducing unwanted and misfolded proteins. Proteasome inhibitors showed anticancer activity in cancer cells resistant to conventional chemotherapeutic drugs. Bortezomib was the first proteasome inhibitor approved to treat refractory multiple myeloma [28]. WFA treatment of cancer cells showed an accumulation of ubiquitinated proteins signifying its effect on proteasome inhibition, associated with increased ER stress, reactive oxygen species, and proapoptotic signaling [29, 30]. Ghosh et al. reported WFA-induced cell death in breast cancer cells by introducing impaired autophagy and unfolded protein response [31]. WFA inhibits chymotrypsin-like activity of a 26S proteasome in PC3 xenografts in nude mice and the PC3 cell line, leading to accretion of proteasome target proteins $\mathrm{p} 27, \mathrm{Bax}$, and $\mathrm{I} \kappa \mathrm{B}-\alpha$ and increase in PARP cleavage proteins inducing apoptosis in prostate cancer cells [32]. Additional docking studies revealed that WFA blocks the chymotrypsin-like activity of a purified rabbit $20 \mathrm{~S}$ proteasome activity by blocking the $\mathrm{N}$ terminal threonine (Thr1) function.

2.3. WFA Inhibits Angiogenesis. Angiogenesis is one of the hallmarks of cancer growth, where vascular endothelial growth factor (VEGF) regulates angiogenesis. The utilization of anti-VEGF therapies has been successful in prolonging survival benefits in multiple cancer types. Increased VEGF expression in prostate tissues and plasma levels has been associated with tumor grade and biochemical and clinical recurrence of prostate cancer $[33,34]$. Anti-VEGF therapy in phase II clinical trials in prostate cancer showed improved relapse-free survival and disease stabilization [35]. However, the phase III results of antiangiogenic treatment in prostate cancer showed toxicity without improving the overall survival [36]. Plant-based compounds are efficient in preventing the formation of new blood vessels in the tumor microenvironment; hence, several natural compounds such as curcumin, resveratrol, and thymoquinone have been studied as potential antiangiogenic drugs [37-40]. In human umbilical vein endothelial cells (HUVECs), WFA showed an antiangiogenic effect by inhibiting HUVEC sprouting in the threedimensional collagen-I matrix [41]. Similarly, WFA deters VEGF-mediated tube formation by HUVECs and binds to vimentin and intermediate filament protein to inhibit tumor angiogenesis $[42,43]$.

2.4. Effect of WFA on Tumor Growth in Mice. In prostate mouse models (TRAMP: transgenic adenocarcinoma of mouse prostate and Pten-knockout) with spontaneous tumor development, oral administration of WFA showed a significant decrease in prostate tumor growth. TRAMP mouse studies revealed that WFA inhibited AKT and pAKT expression and activated Foxo3a-Par-4-induced tumor cell death in mice [44]. Foxo3a works upstream of Par-4 signaling and is essential for apoptosis induction in castration-resistant prostate cancer (CRPC) cells. Deleting the Foxo3a binding site on the Par-4 promoter inhibits Foxo3a-Par-4 interaction and Par-4 activation, suggesting the positive role of Foxo3a in Par-4 stimulation and apoptosis [45]. In Pten-knockout 
TABLE 1: Nonimmune molecular targets of WFA and Withania somnifera (WS) root extract in prostate cancer.

\begin{tabular}{|c|c|c|}
\hline Compound type & Model system & Molecular targets \\
\hline Withaferin A & LNCaP, PC3, and 22RV1 cell lines & $\begin{array}{l}\text { Induces cytoprotective autophagy by increasing } \\
\text { GABARAPL1 (ATG8L) expression [11] }\end{array}$ \\
\hline Withaferin A & PC3 cell line & $\begin{array}{l}\text { Induction of prostate apoptosis response-4- } \\
\text { (Par-4-) dependent apoptosis [14] }\end{array}$ \\
\hline Withaferin A & PC3 and DU145 cell lines & $\begin{array}{c}\text { Upregulation of Aurora B, phosphor histone H3, } \\
\text { and phospho-Wee-1 expression and downregulation } \\
\text { of cyclins (A2, B1, and E2) and phospho-Chk1 (Ser345), } \\
\text { Chk2 (Thr68), and Cdc2 (Tyr15) [22] }\end{array}$ \\
\hline Withaferin A & Cell lines PC3, DU145, and LNCaP & $\begin{array}{l}\text { Disrupt vimentin cytoskeleton by induction of ROS } \\
\text { and c-Fos expression and suppression of c-FLIP(L) [23] }\end{array}$ \\
\hline Withaferin A & $\begin{array}{l}\text { LNCaP cells and PC3 xenografts in } \\
\text { nude mice, i.p. injection with } 4 \text { or } \\
\qquad 8 \mathrm{mg} / \mathrm{kg} / \text { day for } 24 \text { days }\end{array}$ & $\begin{array}{l}\text { Target } \beta 5 \text { subunit and inhibition of chymotrypsin-like } \\
\text { activity in vivo tumors of PC3 xenografts } \\
\text { with an accumulation of proteasome target } \\
\text { proteins (p27, Bax, and } \mathrm{I} \kappa \mathrm{B}-\alpha \text { ) and decreased } \\
\text { AR protein in in vitro LNCaP cell line [32] }\end{array}$ \\
\hline Withaferin A & $\begin{array}{l}\text { TRAMP model, oral gavage of } \\
55 \mathrm{mg} / \mathrm{kg}\end{array}$ & $\begin{array}{c}\text { Prevent prostate adenocarcinoma, inhibit AKT signaling, } \\
\text { and activate Foxo3a-Par- } 4 \text {-induced cell death and } \\
\text { EMT markers (vimentin, } \beta \text {-catenin, and snail and } \\
\text { upregulate E-cadherin) }[44]\end{array}$ \\
\hline Withaferin A & $\begin{array}{l}\text { Pten-KO mice, oral gavage with } 3 \text { or } \\
5 \mathrm{mg} / \mathrm{kg}\end{array}$ & $\begin{array}{l}\text { Inhibit primary tumor growth and lung metastasis, } \\
\text { downregulation of pAKT-mediated EMT markers [46] }\end{array}$ \\
\hline $\begin{array}{l}\text { Withaferin A and ethanol extract } \\
\text { of WS root }\end{array}$ & LNCaP and 22RV1 cell lines & $\begin{array}{l}\text { Inhibits fatty acid synthesis by decreasing fatty } \\
\text { acid metabolism enzymes: acetyl-CoA carboxylase } 1 \text {, } \\
\text { ATP citrate lyase, carnitine palmitoyltransferase } 1 \mathrm{~A}, \\
\text { and fatty acid synthase with a decrease in c-Myc } \\
\text { and pAKT [49] }\end{array}$ \\
\hline WS root extract & PC3 cell line & $\begin{array}{c}\text { Inhibit expression levels of cyclooxygenase-2 } \\
\text { and interleukin-8 [50] }\end{array}$ \\
\hline
\end{tabular}

AR: androgen receptor; EMT: epithelial-to-mesenchymal transition; i.p.: intraperitoneal; LPS: lipopolysaccharides; PARP: poly (ADP-ribose) polymerase; ROS: reactive oxygen species; TRAMP: transgenic adenocarcinoma of mouse prostate.

mice, WFA treatment obliterated lung metastasis of prostate cancer and was associated with a decrease in epithelial-tomesenchymal transition markers (N-cadherin and $\beta$ catenin) [46]. A significant reduction in tumor growth with subcutaneous xenografts of ovarian cancer and glioblastoma cells in nude mice also demonstrated the therapeutic efficacy of WFA $[47,48]$.

Thus, WFA showed pleiotropic functions and exerted its potential anticancer effect via targeting multiple molecular pathways to incapacitate the cancer cell activities. Table 1 summarizes the nonimmune molecular targets of WFA in prostate cancer.

\section{Effect of WFA in Targeting Inflammation via Inflammasomes}

The association of chronic inflammation in cancer development and progression is well-established [51-54]. Therefore, studies are focused on targeting inflammatory cytokines to understand the immunobiology of prostate cancer [55-58]. Mechanistically, a diverse array of signals such as inflammasomes, toll-like receptors (TLRs), and transcription factors regulate the secretion of inflammatory cytokines. Upregulated inflammasome activity is correlated with various cancer types, including gastric cancer, breast cancer, prostate cancer, and brain tumor [59-61]. TLRs, a family of pattern recognition receptors, drive inflammation via activating NF- $\kappa \mathrm{B}$ signaling to promote prostate cancer development $[62,63]$. Indeed, the transcriptional activity of NF- $\kappa \mathrm{B}$ and associated signaling pathways are implicated in multiple disease types and have been a prime target for pharmacological interventions [64]. Nuclear translocation of NF- $\kappa$ B in prostate cancer cells was associated with biochemical recurrence and bone metastatic prostate cancer development. In a retrospective study using a multi-institutional cohort, Grosset et al. showed that an increased level of nuclear NF- $\kappa \mathrm{B}$ p 65 might serve as a prognostic marker for prostate cancer [65]. The study performed tissue microarrays of radical prostatectomy specimens from two independent cohorts $(n=250$ and $n=$ 1262) of treatment-naïve prostate cancer patients collected at multiple centers. The association of p65 nuclear expression and prostate cancer outcome was evaluated by immunohistochemistry. Multivariate analysis revealed that p65 nuclear frequency in prostate cancer cells was an independent predictor of biochemical recurrence and may help identify high-risk prostate cancer patients. In another study, a similar analysis of $\mathrm{NK}-\kappa \mathrm{B}$ p 65 in 105 prostate tissue specimens showed nuclear translocation of p65 expression during the 
transition of disease from prostatic intraepithelial neoplasia to prostate cancer [66]. Multivariate analysis showed that preoperative PSA level, Gleason score, and nuclear $\mathrm{KF}-\kappa \mathrm{B}$ p65 translocation were independent predictors of biochemical relapse. Thus, blocking NF- $\kappa \mathrm{B}$ activation is a promising target for modifying the cancer-associated inflammation and immune activation signaling.

Dietary agents are considered potent inhibitors of the $\mathrm{NF}-\kappa \mathrm{B}$ signaling pathway and reduce cancer-associated inflammation [67]. For example, curcumin, a dietary supplement from turmeric spice, and ginger extract showed anticancer and anti-inflammatory effects by inhibiting NF- $\kappa \mathrm{B}$ activity [68-71]. Lycopene blocks NF- $\kappa \mathrm{B}$ signaling to inhibit prostate cancer cell growth in vitro and reduces in vivo prostate cancer growth in mice $[72,73]$. In a chronic prostatitis rat model, the intragastric lycopene administration $(20 \mathrm{mg} / \mathrm{kg}$, daily for four weeks) reduces inflammation in the prostate and the serum level of IL- $1 \beta$, TNF- $\alpha$, IL-2, and IL-6 cytokines [74]. This anti-inflammatory effect of lycopene was attributed to NF- $\kappa \mathrm{B}$ inhibition in the prostate tissues [74]. Resveratrol isolated from grapes is also one of the potent inhibitors of NF- $\kappa \mathrm{B}$ and leads to anti-inflammatory and antitumor effects [75]. Likewise, ursolic acid derived from berries, leaves, and fruits is a pentacyclic triterpenoid with anti-inflammatory effects by inhibiting NF- $\kappa \mathrm{B}$ signaling through IKK $\alpha$ and p65 phosphorylation [76]. WFA inhibits $\mathrm{NF}-\kappa \mathrm{B}$ activation by blocking Akt to reduce nitric oxide and inducible nitric oxide synthase expression in RAW 264.7 macrophage cell line regulating inflammatory process [77], suggesting that WFA has alternative pathways to regulate $\mathrm{NF}-\kappa \mathrm{B}$ activation. Based on protein array analysis, it has been demonstrated that WFA regulates multiple cytokines in LPS-induced THP-1 cells. In silico studies showed that the downregulated cytokines possess a common regulatory factor NF- $\kappa \mathrm{B}$ and that WFA blocked the nuclear translocation of NF- $\kappa \mathrm{B}$ [78]. The root extract of $W$. somnifera is also known for its antioxidant, anti-inflammatory, and immunomodulatory activities $[79,80]$. Culture supernatant from the LPS-primed, $W$. somnifera root extract- $(0.05-0.4 \mathrm{mg} / \mathrm{ml})$ treated human PBMCs, and THP-1 cells showed decreased TNF- $\alpha$, IL- $1 \beta$, and IL- 6 levels as measured by ELISA [79]. However, further discussions are limited in this article since a recent review described a general perspective of WFA in chronic inflammation [81]. These studies suggest that the medicinal plant product regulating NF- $\kappa \mathrm{B}$ activity can alter inflammatory profiles to benefit the host, targeting inflammation-associated cancer.

Scientific advancement in understanding the regulation of inflammation also suggests the role of the inflammasome signaling complex in the activation of inflammationassociated responses. The inflammasome consists of a nucleotide-binding domain, leucine-rich repeat, and pyrin domain (NRLP), along with caspase activation and recruitment domain (ASC) and procaspase-1. Microbe-associated molecular patterns (MAMPs), danger-associated molecular patterns (DAMPs), pattern-recognition receptors (PRRs), and various pathogens activate the inflammasome. Once activated, the inflammasome recruits the ASC and procaspase- 1 producing an active form of caspase- 1 , which cleaves the proform of interleukin- (IL-) $1 \beta$ and IL-18 to release mature IL- $1 \beta$ and IL-18 associated with various biological activities $[60,61]$.

Although limited information is available on the effect of WFA in regulating inflammasomes, a mouse model of chronic pancreatitis showed that WFA blocks ER stress and the NLRP3 inflammasome to prevent pancreatitis progression [82]. WFA inhibits Helicobacter pylori-induced IL- $1 \beta$ and NLRP3 inflammasome signaling molecules in bone marrow-derived dendritic cells and macrophages, indicating the preventive and therapeutic effect of WFA in H. pylori infection-associated cancers [83]. Pretreatment of mice with WFA inhibited ovalbumin-induced lung injury and fibrosis progression. This preventive effect was attributed to decreased inflammatory cell infiltration in the lungs and a correspondingly low level of proinflammatory cytokines and reduced NLRP3 inflammasome activation [84]. WFA disintegrates the NLRP3 inflammasome complex reducing downstream signaling products IL- $1 \beta$ and IL-18. WFA blocks the NF- $\kappa \mathrm{B}$ activity altering the level of multiple genes associated with $\mathrm{NF}-\kappa \mathrm{B}$ regulation and inhibits inflammasome activity suppressing inflammatory cytokine network [78]. Growing evidence suggests that the inflammasomes play a key role in inflammation-associated diseases, including cancer, and emerges as a game-changer in understanding how inflammation affects the immunobiology of cancer [61]. Therefore, the mechanism of WFA in the regulation of inflammasome activity warrants further investigation.

\section{Effect of WFA on Immune Cell Regulation and Antitumor Immunity}

Medicinal herbs have long been recognized as a way to increase immune system activation. However, the mechanism(s) regulating specific immune cells' functional activation by these herbs, including WFA, remains mostly unknown. Barua et al. showed that $W$. somnifera treatment induces natural killer (NK) cell activation [85], an essential component of the innate immune response to tumors, and actively eliminates early neoplastic cells. NK cells kill tumor targets on contact using perforin (a cytolytic protein) and granzyme (protease family member) machinery [86, 87]. Similarly, IFN- $\gamma$ is a key cytokine produced by NK cells and $\mathrm{T}$ lymphocytes and facilitates the antitumor response. Malik et al. showed that herbal formulation of $W$. somnifera induced Th1 immunity in tumor-bearing mice as measured by the secretion of IFN- $\gamma$ and IL-12 and increased proliferation of $\mathrm{CD}^{+}, \mathrm{CD}^{+} \mathrm{T}$ cells, and NK cells [88]. Following $W$. somnifera supplement, hens susceptible to ovarian cancer displayed a significant reduction in tumor development associated with increased NK cell population [85].

Similarly, oral administration of $W$. somnifera extract (400 mg/kg body weight) once a week for four weeks treating azoxymethane-induced colon cancer in Swiss albino mice increased the number and functional activity of immune cells [89]. NMITLI 101R, a chemotype of W. somnifera, generated humoral and cellular immune responses in Balb/c mice as measured by a high number of antibody-producing cells. The cytokine response remained polarized to the Th1 type 
DMSO (Control)
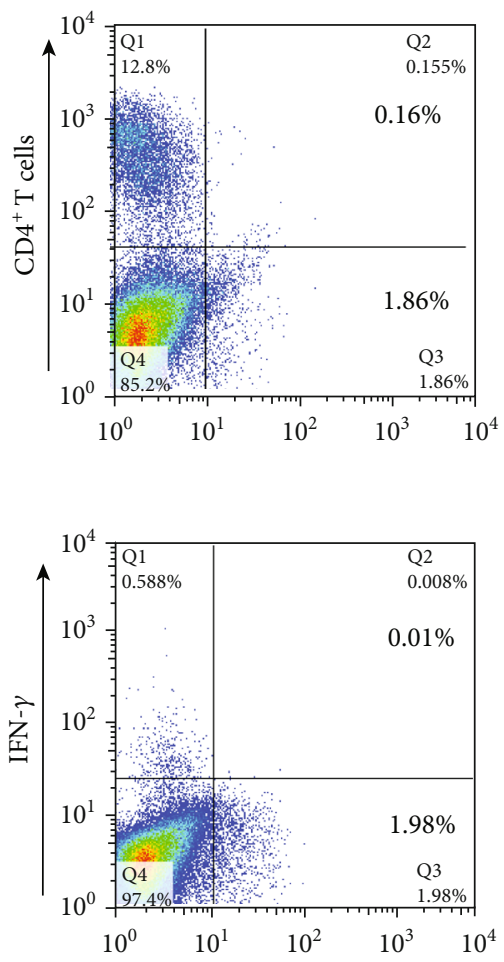

WFA: $2.5 \mu \mathrm{M}$

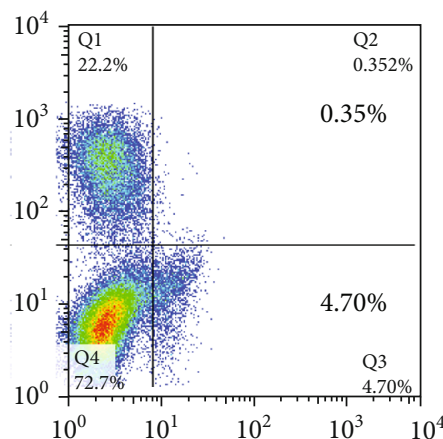

(a)

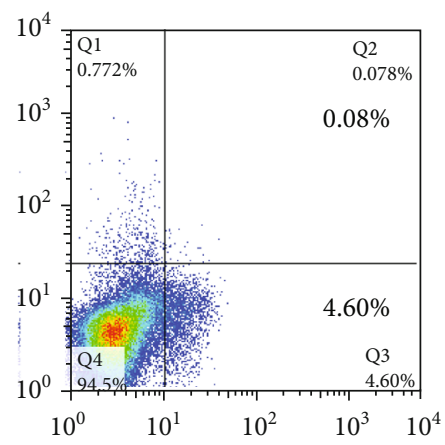

WFA: $10.0 \mu \mathrm{M}$
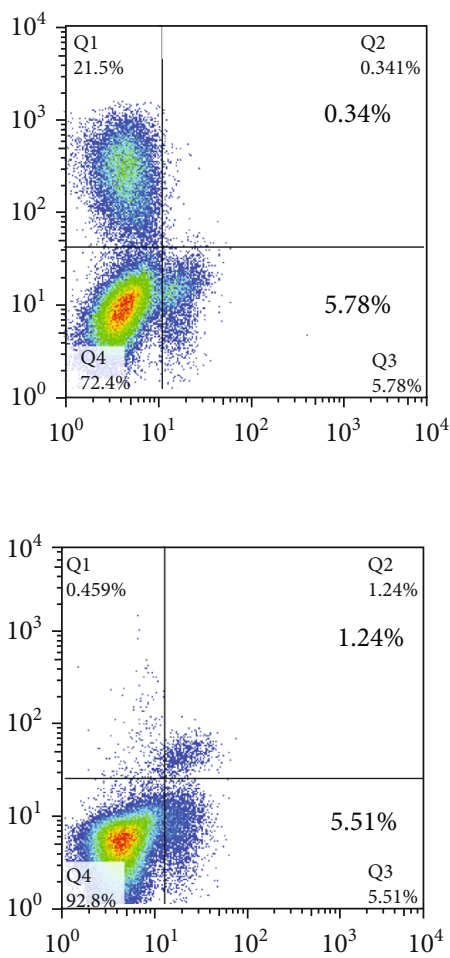

NK cells
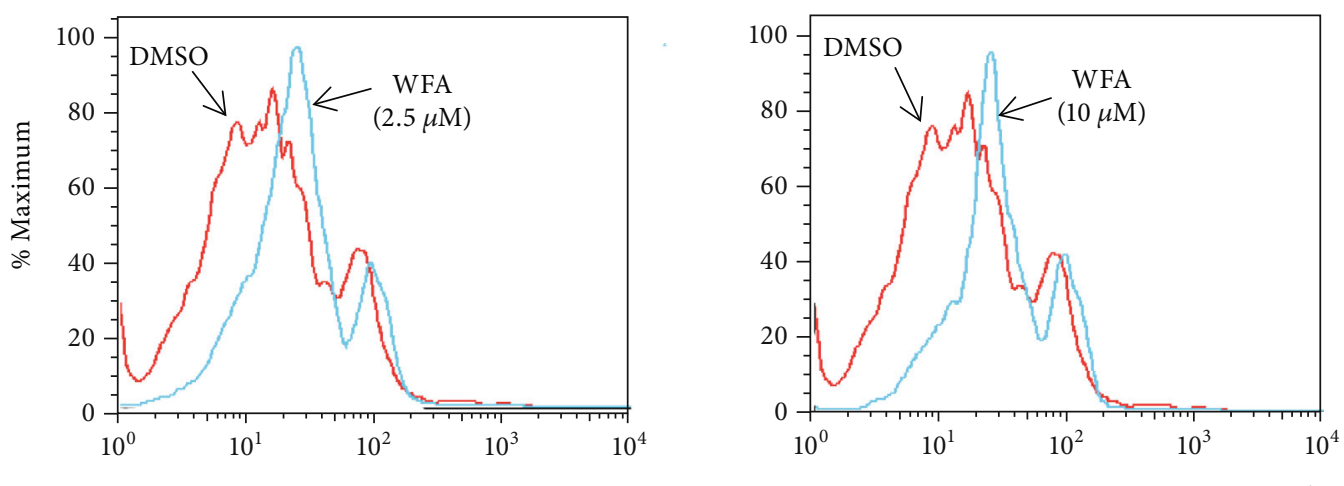

Fluorescence intensity (Perforin)

(b)

FIGURE 1: (a) WFA-induced frequency and (b) function (increase in IFN- $\gamma$ and perforin) of NK cells examined by flow cytometry gated on CD8negative T cells. Splenocytes from the naïve C57BL/6 mice were homogenized into a single-cell suspension, seeded at a density of $5 \times 10^{6}$ cells/well supplemented with cytokine IL-2 $(10 \mathrm{U} / \mathrm{ml})$ in 24-well plates, and incubated overnight in the presence or absence of WFA and DMSO as control.

with increased levels of IFN- $\gamma$ [90]. In parasite-infected hamsters, the chemotype NMITLI 101R surges antileishmanial drug efficacy by generating strong IFN- $\gamma$ - and IL-12mediated immune responses while suppressing the Th2 cytokines (IL-4, IL-10, and TGF- $\beta$ ) [91].

The present team also examined the effect of WFA and found that the WFA augments the quality and quantity of $\mathrm{NK}$ cell function measured by intracellular cytokine staining for IFN- $\gamma$ and perforin. The number of NK cells producing IFN- $\gamma$ and perforin was significantly higher in WFA-treated splenocytes than in the DMSO control (Figures 1(a) and 1(b)).

To further study the role of WFA-induced NK cells in antitumor immunity, the authors used RM1 mouse prostate tumor cells (syngeneic to C57BL/6 mice). RM1 cells express a low level of MHC I and are sensitive to NK cell killing [92]. Intraperitoneal administration of WFA $(8 \mathrm{mg} / \mathrm{kg} / \mathrm{BW})$ significantly $(p=0.04)$ inhibited the growth of established prostate tumors (Figures 2(a) and 2(b)). To follow the prolonged therapeutic effect of WFA, these experimental mice 


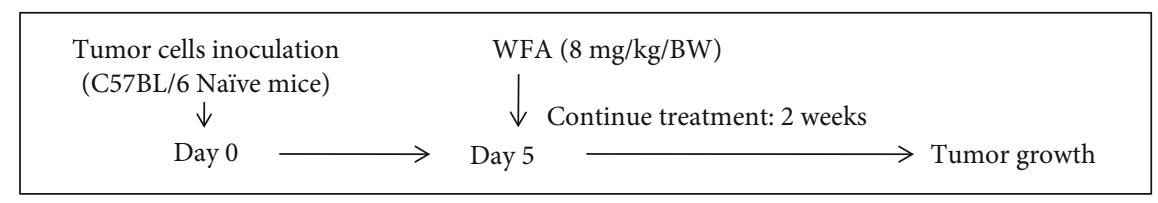

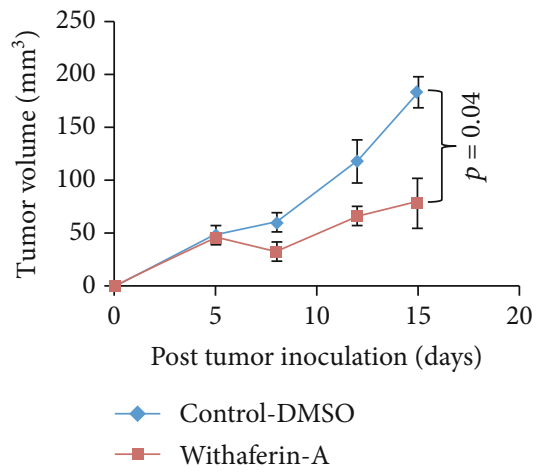

(a)

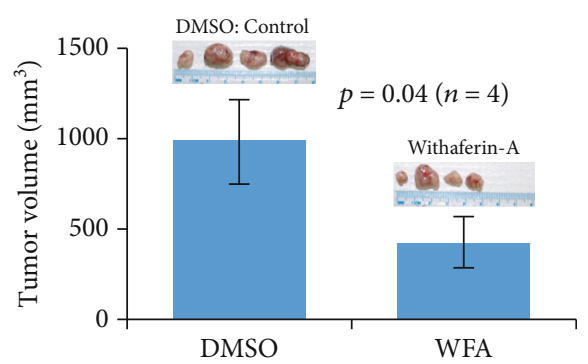

(b)

FIGURE 2: (a) WFA-induced activation of anticancer activity inhibiting the growth of established tumors in mice inoculated with RM1 mouse prostate tumor cells; (b) tumor volume on day 29. Six-to-seven-week-old, naive C57BL/6 mice were inoculated subcutaneously with RM1 tumor cells $\left(5 \times 10^{5}\right)$. Starting on day five following tumor inoculations, a group of mice (4 mice/group) received an intraperitoneal (i.p.) injection of WFA $(8 \mathrm{mg} / \mathrm{kg} / \mathrm{BW})$ five days per week for two weeks. DMSO serves as the control at the same level $(v / v)$ as for WFA treatment groups.

were left untreated for ten additional days. It appeared that the developed immune response following WFA treatment maintained the reduced tumor growth (Figure 2(b)). These observations indicate that the WFA induces NK cell activation, thereby augmenting antitumor immunity to inhibit the development and progression of prostate tumors; however, these preliminary studies need further validation.

Both mouse and human studies further support the role of WFA in the induction of NK cells. Balb/c mice were given an i.p. injection of $W$. somnifera root extract with $20 \mathrm{mg} / \mathrm{do}-$ se/mouse for 5 consecutive days [93]. Mouse splenocytes were harvested 24 hours after the last injection, and were used as effectors against NK-sensitive K562 cells in a chromium release assay to measure the cytotoxic activity of $\mathrm{NK}$ cells. Splenocytes from WFA-treated mice showed $\sim 53 \%$ higher cytotoxicity than splenocytes from vehicle-treated control mice. An observational study in humans also supports WFA-mediated NK cell regulation. Five human subjects consumed $6 \mathrm{ml}$ of $W$. somnifera root extract with cow's milk (8 ounces) twice daily for four days. Peripheral blood sample analysis revealed a significant increase in mean fluorescence intensity of CD $4^{+} \mathrm{T}(4.2$-fold; $p<0.05)$ cells and NK cell activation (3.2-fold; $p<0.01)$ after four days compared to the baseline [94].

Although therapeutic targeting of prostate cancer has evolved remarkably, one significant challenge remains that prostate tumors are enriched with immune suppressor cells (myeloid-derived suppressor cells (MDSCs), M2 macrophages, and T-regulatory cells), hampering the benefits of therapeutic regimens $[95,96]$. Sinha and OstrandRosenberg reported that administration of WFA in tumorbearing mice with $4 \mathrm{~T} 1$ cells significantly reduces the tumor burden, decreases the number of MDSCs and reactive oxygen species (ROS), and suppresses the protumor cytokine IL-10 by the MDSCs and macrophages [97]. WFA was orally administered in mice at different doses ( $1-8 \mathrm{mg} / \mathrm{kg}$ body weight) every other day for the study duration and found that $1 \mathrm{mg} / \mathrm{kg}$ dose of WFA was efficient in delaying tumor growth and suppressing MDSCs in the circulation. In vitro analysis revealed that $1 \mu \mathrm{g} / \mathrm{ml}$ of WFA reduces ROS (measured by $\mathrm{H}_{2} \mathrm{O}_{2}$ ) production by $>50 \%$ in MDSCs while $1 \mu \mathrm{g} / \mathrm{ml}$ treatment dose of withanolide $\mathrm{A}$ and $W$. somnifera root extract was ineffective. The dose of $W$. somnifera root extract was increased to $166.7 \mu \mathrm{g} / \mathrm{ml}$ to achieve a concentration equal to $1 \mu \mathrm{g} / \mathrm{ml}$ of WFA for complete inhibition of $\mathrm{H}_{2} \mathrm{O}_{2}$ production in MDSCs. Withaferin A also inhibited the secretion of proinflammatory cytokines TNF- $\alpha$, IL-6, and IL-12 from macrophages suggesting the role of WFA as a potent inhibitor of proinflammatory mediators [97]. This study corroborates with previous annotations that the use of WFA polarized the immunity towards the Th1 type augmenting antitumor immunity $[88,90,91]$. Thus, the stimulation of NK cell activity and decreased immune suppressor cells provide a novel opportunity to test the immunotherapeutics of WFA in prostate cancer studies.

When boosting the body's immune system for the prevention or treatment of cancer, one of the critical components is to activate the antigen-presenting cells (APCs) such as macrophages and dendritic cells (DCs). Induction of Th1-type immune response is associated with tumor suppression, while Th2 immune responses are tumor-promoting. It is noticeable that WFA treatment polarized the immune response towards the Th1 type of immunity, increasing IFN- $\gamma$ and IL-12 while decreasing Th2 type-associated IL-4, IL-10, and TGF- $\beta$ cytokines. Thus, WFA injection of mice potentially activates APCs to release IL-12 and TNF- $\alpha$ cytokines activating NK cell functions; however, such a hypothesis needs further validation. 


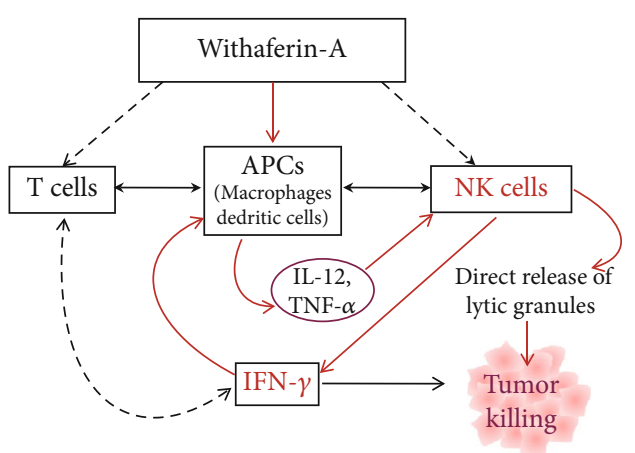

FIGURE 3: WFA triggers immunostimulatory cascade associated with NK cell regulation and antitumor immunity.

Figure 3 describes a schematic presentation for the potential mechanism of WFA-induced immune cell activation.

\section{Clinical Potential of WFA}

A PubMed search for clinical trials using the term "Withania somnifera" yielded few clinical studies testing the impact of $W$. somnifera. Most of these studies consumed root extracts of $W$. somnifera alone or combined with other therapeutic drugs. The published clinical studies demonstrated the safety profile and efficacy of root preparation in various conditions, including chronic stress and anxiety [98-100], schizophrenia [101, 102], memory and cognitive improvement [103], obsessive-compulsive disorder [104], and subclinical hypothyroidism [105]. However, clinical studies testing the impact of $W$. somnifera directly in cancer patients are minimal. In an open-label prospective nonrandomized comparative trial on 100 breast cancer patients in all stages who received chemotherapy with or without oral $W$. somnifera, the use of $W$. somnifera showed an improvement in the quality-of-life and fatigue scores [106]. A phase I study of WFA in advanced-stage high-grade osteosarcoma patients also demonstrated that WFA was safe and well-tolerated up to $4800 \mathrm{mg}$ per day [107]. Surprisingly, no clinical study has tested the efficacy of WFA for prostate cancer.

\section{Conclusions and Future Perspectives}

Withania somnifera establishes various health benefits, including antistress, anti-inflammatory, anticancer, and immunostimulatory properties. Indeed, administration of WFA and its impact on tumor growth inhibition in TRAMP and Pten-knockout prostate cancer mouse models with functional immune systems indicate enhanced antitumor immunity. However, to further understand the role of WFA in regulating the immunobiology of prostate cancer, detailed studies are needed to analyze the infiltrating immune cells in these tumors. Many prostate cancers are organ-confined at the time of diagnosis, and some patients prefer to remain under active surveillance. Since WFA-induces Th1-type responses and NK cell activation, the slow-growing nature of some prostate cancers may provide an opportunity for immune manipulation at early prostate cancer stages and may represent a paradigm for the use of WFA for cancer- related or chemotherapy-induced fatigue, cancer prevention, and therapeutic efficacy. WFA usage combined with standard treatment regimens may help induce both innate and adaptive immune system arms to achieve greater therapeutic efficacy to target prostate cancer. The preclinical observations suggest pluripotent functions of WFA targeting prostate cancer; therefore, more extensive randomized clinical studies are needed to establish the safety profile of the WFA compound.

\section{Data Availability}

The additional experimental data used to support the findings of this study are included within the article.

\section{Conflicts of Interest}

The authors have no financial conflict of interest.

\section{Authors' Contributions}

Conceptualization was performed by D.K. and S.D.; writing and original draft preparation were performed by D.K., S.D., M.S., and A.N.; writing, reviewing, and editing were performed by D.K., S.D., M.S., and A.N.; supervision was performed by D.K.; project administration was performed by D.K.; D.K was responsible for funding acquisition. All authors agreed and approved the final manuscript.

\section{Acknowledgments}

DK's research is supported by the National Institutes of Health (CA204786). A part of these studies was initiated during the National Institutes of Health (NIH)/NCI funding (CA169453) to DK.

\section{References}

[1] M. B. Culp, I. Soerjomataram, J. A. Efstathiou, F. Bray, and A. Jemal, "Recent global patterns in prostate cancer incidence and mortality rates," European Urology, vol. 77, no. 1, pp. 3852, 2020.

[2] D. J. Newman and G. M. Cragg, "Natural products as sources of new drugs over the nearly four decades from 01/1981 to 09/2019," Journal of Natural Products, vol. 83, no. 3, pp. 770-803, 2020.

[3] M. Matsushita, K. Fujita, and N. Nonomura, "Influence of diet and nutrition on prostate cancer," International Journal of Molecular Sciences, vol. 21, no. 4, p. 1447, 2020.

[4] P. H. Lin, W. Aronson, and S. J. Freedland, "Nutrition, dietary interventions and prostate cancer: the latest evidence," BMC Medicine, vol. 13, no. 3, 2015.

[5] E. M. Schlag and M. S. McIntosh, "Ginsenoside content and variation among and within American ginseng (Panax quinquefolius L.) populations," Phytochemistry, vol. 67, no. 14, pp. 1510-1519, 2006.

[6] M. Elsakka, E. Grigorescu, U. Stanescu, U. Stanescu, and V. Dorneanu, "New data referring to chemistry of Withania somnifera species," Revista Medico-Chirurgicală a Societății de Medici şi Naturalişti din Iaşi, vol. 94, no. 2, pp. 385-387, 1990. 
[7] E. P. Sabina, S. Chandal, and M. K. Rasool, "Inhibition of monosodium urate crystal-induced inflammation by withaferin a," Journal of Pharmacy \& Pharmaceutical Sciences, vol. 11, no. 4, pp. 46-55, 2008.

[8] R. Munagala, H. Kausar, C. Munjal, and R. C. Gupta, "Withaferin $\mathrm{A}$ induces p53-dependent apoptosis by repression of HPV oncogenes and upregulation of tumor suppressor proteins in human cervical cancer cells," Carcinogenesis, vol. 32, no. 11, pp. 1697-1705, 2011.

[9] S. D. Stan, E. R. Hahm, R. Warin, and S. V. Singh, "Withaferin A causes FOXO3a- and Bim-dependent apoptosis and inhibits growth of human breast cancer cells in vivo," Cancer Research, vol. 68, no. 18, pp. 7661-7669, 2008.

[10] V. S. Sivasankarapillai, R. Madhu Kumar Nair, A. Rahdar et al., "Overview of the anticancer activity of withaferin A, an active constituent of the Indian ginseng Withania somnifera," Environmental Science and Pollution Research International, vol. 27, no. 21, pp. 26025-26035, 2020.

[11] E. R. Hahm, S. H. Kim, K. B. Singh, K. Singh, and S. V. Singh, "A comprehensive review and perspective on anticancer mechanisms of withaferin A in breast cancer," Cancer Prevention Research (Philadelphia, Pa.), vol. 13, no. 9, pp. 721-734, 2020.

[12] B. Hassannia, E. Logie, P. Vandenabeele, T. Vanden Berghe, W. Vanden Berghe, and A. Withaferin, "Withaferin A: from ayurvedic folk medicine to preclinical anti-cancer drug," Biochemical Pharmacology, vol. 173, article 113602, 2020.

[13] X. Zhang, A. K. Samadi, K. F. Roby, B. Timmermann, and M. S. Cohen, "Inhibition of cell growth and induction of apoptosis in ovarian carcinoma cell lines CaOV 3 and SKOV 3 by natural withanolide withaferin A," Gynecologic Oncology, vol. 124, no. 3, pp. 606-612, 2012.

[14] S. Srinivasan, R. S. Ranga, R. Burikhanov, S. S. Han, and D. Chendil, "Par-4-dependent apoptosis by the dietary compound withaferin A in prostate cancer cells," Cancer Research, vol. 67, no. 1, pp. 246-253, 2007.

[15] T. Behl, A. Sharma, L. Sharma et al., "Exploring the multifaceted therapeutic potential of withaferin A and its derivatives," Biomedicines, vol. 8, no. 12, p. 571, 2020.

[16] G. Kim, T. H. Kim, E. H. Hwang, K. T. Chang, J. J. Hong, and J. H. Park, "Withaferin A inhibits the proliferation of gastric cancer cells by inducing G2/M cell cycle arrest and apoptosis," Oncology Letters, vol. 14, no. 1, pp. 416-422, 2017.

[17] H.-W. Chang, R.-N. Li, H.-R. Wang et al., "Withaferin A induces oxidative stress-mediated apoptosis and DNA damage in oral cancer cells," Frontiers in Physiology, vol. 8, p. $634,2017$.

[18] S. D. Stan, Y. Zeng, and S. V. Singh, "Ayurvedic medicine constituent withaferin a causes G2 and M phase cell cycle arrest in human breast cancer cells," Nutrition and Cancer, vol. 60, Supplement 1, pp. 51-60, 2008.

[19] Q. Tang, L. Ren, J. Liu et al., "Withaferin A triggers G2/M arrest and intrinsic apoptosis in glioblastoma cells via ATF4-ATF3-CHOP axis," Cell Proliferation, vol. 53, no. 1, article e12706, 2020.

[20] S. Okamoto, T. Tsujioka, S. I. Suemori et al., "Withaferin A suppresses the growth of myelodysplasia and leukemia cell lines by inhibiting cell cycle progression," Cancer Science, vol. 107, no. 9, pp. 1302-1314, 2016.

[21] A. Batra and E. Winquist, "Emerging cell cycle inhibitors for treating metastatic castration-resistant prostate cancer,"
Expert Opinion on Emerging Drugs, vol. 23, no. 4, pp. 271282, 2018.

[22] R. V. Roy, S. Suman, T. P. Das, J. E. Luevano, and C. Damodaran, "Withaferin A, a steroidal lactone from Withania somnifera, induces mitotic catastrophe and growth arrest in prostate cancer cells," Journal of Natural Products, vol. 76, no. 10, pp. 1909-1915, 2013.

[23] Y. Nishikawa, D. Okuzaki, K. Fukushima et al., "Withaferin A induces cell death selectively in androgen-independent prostate cancer cells but not in normal fibroblast cells," PLoS One, vol. 10, no. 7, article e0134137, 2015.

[24] B. Y. Choi and B.-W. Kim, "Withaferin-A inhibits colon cancer cell growth by blocking STAT3 transcriptional activity," Journal of Cancer Prevention, vol. 20, no. 3, pp. 185-192, 2015.

[25] S. S. Kakar, C. A. Worth, Z. Wang, K. Carter, M. Ratajczak, and P. Gunjal, "DOXIL when combined with withaferin A (WFA) targets ALDH1 positive cancer stem cells in ovarian cancer," Journal of Cancer Stem Cell Research, vol. 4, no. 2, p. 1, 2016.

[26] T.-J. Lee, H. J. Um, J.-W. Park, K. S. Choi, and T. K. Kwon, "Withaferin A sensitizes TRAIL-induced apoptosis through reactive oxygen species-mediated up-regulation of death receptor 5 and down-regulation of c-FLIP," Free Radical Biology and Medicine, vol. 46, no. 12, pp. 1639-1649, 2009.

[27] A. Nagalingam, P. Kuppusamy, S. V. Singh, D. Sharma, and N. K. Saxena, "Mechanistic elucidation of the antitumor properties of withaferin A in breast cancer," Cancer Research, vol. 74, no. 9, pp. 2617-2629, 2014.

[28] Q. Ping Dou, “A Zonder J. Overview of proteasome inhibitorbased anti-cancer therapies: perspective on bortezomib and second generation proteasome inhibitors versus future generation inhibitors of ubiquitin-proteasome system," Current Cancer Drug Targets, vol. 14, no. 6, pp. 517-536, 2014.

[29] M. Ri, "Endoplasmic-reticulum stress pathway-associated mechanisms of action of proteasome inhibitors in multiple myeloma," International Journal of Hematology, vol. 104, no. 3, pp. 273-280, 2016.

[30] M. J. Choi, E. J. Park, K. J. Min, J.-W. Park, and T. K. Kwon, "Endoplasmic reticulum stress mediates withaferin Ainduced apoptosis in human renal carcinoma cells," Toxicology In Vitro, vol. 25, no. 3, pp. 692-698, 2011.

[31] K. Ghosh, S. De, S. Mukherjee, S. Das, A. N. Ghosh, and S. B. Sengupta, "Withaferin A induced impaired autophagy and unfolded protein response in human breast cancer cell-lines MCF-7 and MDA-MB-231," Toxicology In Vitro, vol. 44, pp. 330-338, 2017.

[32] H. Yang, G. Shi, and Q. P. Dou, "The tumor proteasome is a primary target for the natural anticancer compound withaferin A isolated from "Indian winter cherry"," Molecular Pharmacology, vol. 71, no. 2, pp. 426-437, 2007.

[33] J. L. Duque, K. R. Loughlin, R. M. Adam, P. Kantoff, E. Mazzucchi, and M. R. Freeman, "Measurement of plasma levels of vascular endothelial growth factor in prostate cancer patients: relationship with clinical stage, Gleason score, prostate volume, and serum prostate-specific antigen," Clinics (São Paulo, Brazil), vol. 61, no. 5, pp. 401-408, 2006.

[34] Y. Nordby, S. Andersen, E. Richardsen et al., "Stromal expression of VEGF-A and VEGFR-2 in prostate tissue is associated with biochemical and clinical recurrence after radical prostatectomy," The Prostate, vol. 75, no. 15, pp. 1682-1693, 2015. 
[35] R. R. McKay, A. J. Zurita, L. Werner et al., "A randomized phase II trial of short-course androgen deprivation therapy with or without bevacizumab for patients with recurrent prostate cancer after definitive local therapy," Journal of Clinical Oncology, vol. 34, no. 16, pp. 1913-1920, 2016.

[36] W. K. Kelly, S. Halabi, M. Carducci et al., "Randomized, double-blind, placebo-controlled phase III trial comparing docetaxel and prednisone with or without bevacizumab in men with metastatic castration-resistant prostate cancer: CALGB 90401," Journal of Clinical Oncology, vol. 30, no. 13, pp. 1534-1540, 2012.

[37] Z. Hoseinkhani, F. Norooznezhad, M. Rastegari-Pouyani, and K. Mansouri, "Medicinal plants extracts with antiangiogenic activity: where is the link?," Advanced Pharmaceutical Bulletin, vol. 10, no. 3, pp. 370-378, 2020.

[38] T. P. Fan, J. C. Yeh, K. W. Leung, P. Y. Yue, and R. N. Wong, "Angiogenesis: from plants to blood vessels," Trends in Pharmacological Sciences, vol. 27, no. 6, pp. 297-309, 2006.

[39] A. E. Gururaj, M. Belakavadi, D. A. Venkatesh, D. Marme, and B. P. Salimath, "Molecular mechanisms of antiangiogenic effect of curcumin," Biochemical and Biophysical Research Communications, vol. 297, no. 4, pp. 934-942, 2002.

[40] W. H. Hu, R. Duan, Y. T. Xia et al., "Binding of resveratrol to vascular endothelial growth factor suppresses angiogenesis by inhibiting the receptor signaling," Journal of Agricultural and Food Chemistry, vol. 67, no. 4, pp. 1127-1137, 2019.

[41] R. Mohan, H. Hammers, P. Bargagna-mohan et al., "Withaferin A is a potent inhibitor of angiogenesis," Angiogenesis, vol. 7, no. 2, pp. 115-122, 2004.

[42] P. Bargagna-Mohan, A. Hamza, Y. E. Kim et al., "The tumor inhibitor and antiangiogenic agent withaferin $\mathrm{A}$ targets the intermediate filament protein vimentin," Chemistry \& Biology, vol. 14, no. 6, pp. 623-634, 2007.

[43] S. Prasanna Kumar, P. Shilpa, and B. P. Salimath, "Withaferin A suppresses the expression of vascular endothelial growth factor in Ehrlich ascites tumor cells via Sp1 transcription factor," Current Trends in Biotechnology and Pharmacy, vol. 3, no. 2, pp. 138-148, 2009.

[44] S. Suman, T. P. Das, J. Moselhy et al., "Oral administration of withaferin A inhibits carcinogenesis of prostate in TRAMP model," Oncotarget, vol. 7, no. 33, pp. 53751-53761, 2016.

[45] T. Das, S. Suman, H. Alatassi, M. Ankem, and C. Damodaran, "Inhibition of AKT promotes FOXO3a-dependent apoptosis in prostate cancer," Cell Death \& Disease, vol. 7, no. 2, pp. e2111-e2111, 2016.

[46] J. Moselhy, S. Suman, M. Alghamdi et al., "Withaferin A inhibits prostate carcinogenesis in a PTEN-deficient mouse model of prostate cancer," Neoplasia, vol. 19, no. 6, pp. 451-459, 2017.

[47] E. Chang, C. Pohling, A. Natarajan et al., "AshwaMAX and withaferin A inhibits gliomas in cellular and murine orthotopic models," Journal of Neuro-Oncology, vol. 126, no. 2, pp. 253-264, 2016.

[48] A. N. Sari, P. Bhargava, J. K. Dhanjal et al., "Combination of withaferin-A and CAPE provides superior anticancer potency: bioinformatics and experimental evidence to their molecular targets and mechanism of action," Cancers (Basel), vol. 12, no. 5, p. 1160, 2020.

[49] S. H. Kim, K. B. Singh, E. R. Hahm, B. L. Lokeshwar, and S. V. Singh, "Withania somnifera root extract inhibits fatty acid synthesis in prostate cancer cells," Journal of Traditional and Complementary Medicine, vol. 10, no. 3, pp. 188-197, 2020.

[50] A. Setty Balakrishnan, A. A. Nathan, M. Kumar, S. Ramamoorthy, and S. K. Ramia Mothilal, "Withania somnifera targets interleukin-8 and cyclooxygenase-2 in human prostate cancer progression," Prostate International, vol. 5, no. 2, pp. 75-83, 2017.

[51] G. Multhoff, M. Molls, and J. Radons, "Chronic inflammation in cancer development," Frontiers in Immunology, vol. 2, p. 98, 2011.

[52] F. R. Greten and S. I. Grivennikov, "Inflammation and cancer: triggers, mechanisms, and consequences," Immunity, vol. 51, no. 1, pp. 27-41, 2019.

[53] L. M. Coussens and Z. Werb, "Inflammation and cancer," Nature, vol. 420, no. 6917, pp. 860-867, 2002.

[54] H. Xu, M.-b. Hu, P.-d. Bai et al., "Proinflammatory cytokines in prostate cancer development and progression promoted by high-fat diet," BioMed Research International, vol. 2015, Article ID 249741, 7 pages, 2015.

[55] T. Hayashi, K. Fujita, S. Nojima et al., "High-fat diet-induced inflammation accelerates prostate cancer growth via IL6 signaling," Clinical Cancer Research, vol. 24, no. 17, pp. 43094318, 2018.

[56] J. S. de Bono, C. Guo, B. Gurel et al., "Prostate carcinogenesis: inflammatory storms," Nature Reviews. Cancer, vol. 20, no. 8, pp. 455-469, 2020.

[57] M. Archer, N. Dogra, and N. Kyprianou, "Inflammation as a driver of prostate cancer metastasis and therapeutic resistance," Cancers (Basel), vol. 12, no. 10, p. 2984, 2020.

[58] D. Karan, J. Holzbeierlein, and J. B. Thrasher, "Macrophage inhibitory cytokine-1: possible bridge molecule of inflammation and prostate cancer," Cancer Research, vol. 69, no. 1, pp. 2-5, 2009.

[59] M. Terlizzi, V. Casolaro, A. Pinto, and R. Sorrentino, "Inflammasome: cancer's friend or foe?," Pharmacology \& Therapeutics, vol. 143, no. 1, pp. 24-33, 2014.

[60] L. Zitvogel, O. Kepp, L. Galluzzi, and G. Kroemer, "Inflammasomes in carcinogenesis and anticancer immune responses," Nature Immunology, vol. 13, no. 4, pp. 343-351, 2012.

[61] D. Karan, "Inflammasomes: emerging central players in cancer immunology and immunotherapy," Frontiers in Immunology, vol. 9, p. 3028, 2018.

[62] S. Zhao, Y. Zhang, Q. Zhang, F. Wang, and D. Zhang, "Tolllike receptors and prostate cancer," Frontiers in Immunology, vol. 5, p. 352, 2014.

[63] T. Ou, M. Lilly, and W. Jiang, "The pathologic role of toll-like receptor 4 in prostate cancer," Frontiers in Immunology, vol. 9, p. 1188, 2018.

[64] B. Eluard, C. Thieblemont, and V. Baud, "NF- $\kappa$ B in the new era of cancer therapy," Trends Cancer, vol. 6, no. 8, pp. 677-687, 2020.

[65] A. A. Grosset, V. Ouellet, C. Caron et al., "Validation of the prognostic value of NF- $\kappa \mathrm{B}$ p65 in prostate cancer: a retrospective study using a large multi-institutional cohort of the Canadian Prostate Cancer Biomarker Network," PLoS Medicine, vol. 16, no. 7, article e1002847, 2019.

[66] J. Domingo-Domenech, B. Mellado, B. Ferrer et al., “Activation of nuclear factor- $\kappa \mathrm{B}$ in human prostate carcinogenesis 
and association to biochemical relapse," British Journal of Cancer, vol. 93, no. 11, pp. 1285-1294, 2005.

[67] H. Khan, H. Ullah, P. Castilho et al., "Targeting NF- $\kappa$ B signaling pathway in cancer by dietary polyphenols," Critical Reviews in Food Science and Nutrition, vol. 60, no. 16, pp. 2790-2800, 2020.

[68] J. U. Marquardt, L. Gomez-Quiroz, L. O. Arreguin Camacho et al., "Curcumin effectively inhibits oncogenic NF- $\kappa$ B signaling and restrains stemness features in liver cancer," Journal of Hepatology, vol. 63, no. 3, pp. 661-669, 2015.

[69] S. Liu, Z. Wang, Z. Hu et al., "Anti-tumor activity of curcumin against androgen-independent prostate cancer cells via inhibition of NF- $\kappa$ B and AP-1 pathway in vitro," Journal of Huazhong University of Science and Technology. Medical Sciences, vol. 31, no. 4, pp. 530-534, 2011.

[70] S. H. Habib, S. Makpol, N. A. Abdul Hamid, S. Das, W. Z. Ngah, and Y. A. Yusof, "Ginger extract (Zingiber officinale) has anti-cancer and anti-inflammatory effects on ethionineinduced hepatoma rats," Clinics (São Paulo, Brazil), vol. 63, no. 6, pp. 807-813, 2008 .

[71] N. A. Abd Wahab, N. H. Lajis, F. Abas, I. Othman, and R. Naidu, "Mechanism of anti-cancer activity of curcumin on androgen-dependent and androgen-independent prostate cancer," Nutrients, vol. 12, no. 3, p. 679, 2020.

[72] L. N. Jiang, Y. B. Liu, and B. H. Li, "Lycopene exerts antiinflammatory effect to inhibit prostate cancer progression," Asian Journal of Andrology, vol. 21, p. 80, 2019.

[73] E. A. Assar, M. C. Vidalle, M. Chopra, and S. Hafizi, "Lycopene acts through inhibition of $\mathrm{I} \kappa \mathrm{B}$ kinase to suppress NF$\kappa \mathrm{B}$ signaling in human prostate and breast cancer cells," Tumour Biology, vol. 37, no. 7, pp. 9375-9385, 2016.

[74] Q. Zhao, F. Yang, L. Meng et al., "Lycopene attenuates chronic prostatitis/chronic pelvic pain syndrome by inhibiting oxidative stress and inflammation via the interaction of NF- $\kappa \mathrm{B}$, MAPKs, and Nrf2 signaling pathways in rats," Andrology, vol. 8, no. 3, pp. 747-755, 2020.

[75] S. C. Gupta, J. H. Kim, R. Kannappan, S. Reuter, P. M. Dougherty, and B. B. Aggarwal, "Role of nuclear factor- $\kappa \mathrm{B}$ mediated inflammatory pathways in cancer-related symptoms and their regulation by nutritional agents," Experimental Biology and Medicine (Maywood, N.J.), vol. 236, no. 6, pp. 658-671, 2011.

[76] S. Shishodia, S. Majumdar, S. Banerjee, and B. B. Aggarwal, "Ursolic acid inhibits nuclear factor-kappaB activation induced by carcinogenic agents through suppression of IkappaBalpha kinase and p65 phosphorylation: correlation with down-regulation of cyclooxygenase 2, matrix metalloproteinase 9, and cyclin D1," Cancer Research, vol. 63, no. 15, pp. 4375-4383, 2003.

[77] J. H. Oh, T. J. Lee, J. W. Park, T. K. Kwon, and A. Withaferin, "Withaferin A inhibits iNOS expression and nitric oxide production by Akt inactivation and down-regulating LPSinduced activity of NF- $\kappa \mathrm{B}$ in RAW 264.7 cells," European Journal of Pharmacology, vol. 599, no. 1-3, pp. 11-17, 2008.

[78] S. Dubey, H. Yoon, M. S. Cohen, P. Nagarkatti, M. Nagarkatti, and D. Karan, "Withaferin A associated differential regulation of inflammatory cytokines," Frontiers in Immunology, vol. 9, p. 195, 2018.

[79] D. B. Naidoo, A. A. Chuturgoon, A. Phulukdaree, K. P. Guruprasad, K. Satyamoorthy, and V. Sewram, "Withania somnifera modulates cancer cachexia associated inflammatory cytokines and cell death in leukaemic THP-1 cells and peripheral blood mononuclear cells (PBMC's)," BMC Complementary and Alternative Medicine, vol. 18, no. 1, p. 126, 2018.

[80] M. Rasool and P. Varalakshmi, "Immunomodulatory role of Withania somnifera root powder on experimental induced inflammation: an in vivo and in vitro study," Vascular Pharmacology, vol. 44, no. 6, pp. 406-410, 2006.

[81] E. Logie and B. W. Vanden, "Tackling chronic inflammation with withanolide phytochemicals-a withaferin A perspective," Antioxidants (Basel), vol. 9, no. 11, 2020.

[82] M. A. Kanak, R. Shahbazov, G. Yoshimatsu, M. F. Levy, M. C. Lawrence, and B. Naziruddin, "A small molecule inhibitor of $\mathrm{NF} \kappa \mathrm{B}$ blocks ER stress and the NLRP3 inflammasome and prevents progression of pancreatitis," Journal of Gastroenterology, vol. 52, no. 3, pp. 352-365, 2017.

[83] J. E. Kim, J. Y. Lee, M. J. Kang et al., "Withaferin A inhibits Helicobacter pylori-induced production of IL- $1 \beta$ in dendritic cells by regulating NF- $\kappa \mathrm{B}$ and NLRP3 inflammasome activation," Immune Network, vol. 15, no. 6, pp. 269-277, 2015.

[84] H. M. Zhao, Z. W. Gao, S. X. Xie, X. Han, and Q. S. Sun, "Withaferin A attenuates ovalbumin induced airway inflammation," Frontiers in Bioscience, vol. 24, pp. 576-596, 2019.

[85] A. Barua, M. J. Bradaric, P. Bitterman et al., "Dietary supplementation of Ashwagandha (Withania somnifera, Dunal) enhances NK cell function in ovarian tumors in the laying hen model of spontaneous ovarian cancer," American Journal of Reproductive Immunology, vol. 70, no. 6, pp. 538-550, 2013.

[86] T. A. Fehniger, S. F. Cai, X. Cao et al., "Acquisition of murine NK cell cytotoxicity requires the translation of a pre- existing pool of granzyme B and perforin mRNAs," Immunity, vol. 26, no. 6, pp. 798-811, 2007.

[87] M. J. Smyth, E. Cretney, J. M. Kelly et al., "Activation of NK cell cytotoxicity," Molecular Immunology, vol. 42, no. 4, pp. 501-510, 2005.

[88] F. Malik, A. Kumar, S. Bhushan et al., "Immune modulation and apoptosis induction: two sides of antitumoural activity of a standardised herbal formulation of Withania somnifera," European Journal of Cancer, vol. 45, no. 8, pp. 1494-1509, 2009.

[89] G. Muralikrishnan, A. K. Dinda, and F. Shakeel, "Immunomodulatory effects of Withania somnifera on azoxymethane induced experimental colon cancer in mice," Immunological Investigations, vol. 39, no. 7, pp. 688-698, 2010.

[90] S. Kushwaha, S. Roy, R. Maity et al., "Chemotypical variations in Withania somnifera lead to differentially modulated immune response in BALB/c mice," Vaccine, vol. 30, no. 6, pp. 1083-1093, 2012.

[91] C. D. Tripathi, P. K. Kushawaha, R. S. Sangwan, C. Mandal, S. Misra-Bhattacharya, and A. Dube, "Withania somnifera chemotype NMITLI 101R significantly increases the efficacy of antileishmanial drugs by generating strong IFN- $\gamma$ and IL-12 mediated immune responses in Leishmania donovani infected hamsters," Phytomedicine, vol. 24, pp. 87-95, 2017.

[92] T. S. Griffith, M. Kawakita, J. Tian et al., "Inhibition of murine prostate tumor growth and activation of immunoregulatory cells with recombinant canarypox viruses," Journal of the National Cancer Institute, vol. 93, no. 13, pp. 998-1007, 2001.

[93] L. Davis and G. Kuttan, "Effect of Withania somnifera on cell mediated immune responses in mice," Journal of 
Experimental \& Clinical Cancer Research, vol. 21, no. 4, pp. 585-590, 2002.

[94] J. Mikolai, A. Erlandsen, A. Murison et al., "In vivo effects of Ashwagandha (Withania somnifera) extract on the activation of lymphocytes," Journal of Alternative and Complementary Medicine, vol. 15, no. 4, pp. 423-430, 2009.

[95] N. Chi, Z. Tan, K. Ma, L. Bao, and Z. Yun, "Increased circulating myeloid-derived suppressor cells correlate with cancer stages, interleukin-8 and -6 in prostate cancer," International Journal of Clinical and Experimental Medicine, vol. 7, no. 10, pp. 3181-3192, 2014.

[96] A. Erlandsson, J. Carlsson, M. Lundholm et al., "M2 macrophages and regulatory T cells in lethal prostate cancer," The Prostate, vol. 79, no. 4, pp. 363-369, 2019.

[97] P. Sinha and S. Ostrand-Rosenberg, "Myeloid-derived suppressor cell function is reduced by withaferin A, a potent and abundant component of Withania somnifera root extract," Cancer Immunology, Immunotherapy, vol. 62, no. 11, pp. 1663-1673, 2013.

[98] S. Fuladi, S. A. Emami, A. H. Mohammadpour, A. Karimani, A. A. Manteghi, and A. Sahebkar, "Assessment of Withania somnifera root extract efficacy in patients with generalized anxiety disorder: a randomized double-blind placebocontrolled trial," Current Clinical Pharmacology, vol. 15, 2020.

[99] A. L. Lopresti, P. D. Drummond, and S. J. Smith, “A randomized, double-blind, placebo-controlled, crossover study examining the hormonal and vitality effects of Ashwagandha (Withania somnifera) in aging, overweight males," American Journal of Men's Health, vol. 13, no. 2, 2019.

[100] A. L. Lopresti, S. J. Smith, H. Malvi, and R. Kodgule, "An investigation into the stress-relieving and pharmacological actions of an Ashwagandha (Withania somnifera) extract: a randomized, double-blind, placebo-controlled study," Medicine (Baltimore), vol. 98, no. 37, article e17186, 2019.

[101] K. N. R. Chengappa, J. S. Brar, J. M. Gannon, and P. J. Schlicht, "Adjunctive use of a standardized extract of Withania somnifera (Ashwagandha) to treat symptom exacerbation in schizophrenia: a randomized, double-blind, placebocontrolled study," The Journal of Clinical Psychiatry, vol. 79, no. 5, 2018.

[102] J. M. Gannon, J. Brar, A. Rai, and K. N. R. Chengappa, "Effects of a standardized extract of Withania somnifera (Ashwagandha) on depression and anxiety symptoms in persons with schizophrenia participating in a randomized, placebo-controlled clinical trial," Annals of Clinical Psychiatry, vol. 31, no. 2, pp. 123-129, 2019.

[103] D. Choudhary, S. Bhattacharyya, and S. Bose, "Efficacy and safety of Ashwagandha (Withania somnifera (L.) Dunal) root extract in improving memory and cognitive functions," Journal of Dietary Supplements, vol. 14, no. 6, pp. 599-612, 2017.

[104] S. P. Jahanbakhsh, A. A. Manteghi, S. A. Emami et al., "Evaluation of the efficacy of Withania somnifera (Ashwagandha) root extract in patients with obsessive-compulsive disorder: a randomized double-blind placebo-controlled trial," Complementary Therapies in Medicine, vol. 27, pp. 25-29, 2016.

[105] A. K. Sharma, I. Basu, and S. Singh, "Efficacy and safety of Ashwagandha root extract in subclinical hypothyroid patients: a double-blind, randomized placebo-controlled trial," Journal of Alternative and Complementary Medicine, vol. 24, no. 3, pp. 243-248, 2018.
[106] B. M. Biswal, S. A. Sulaiman, H. C. Ismail, H. Zakaria, and K. I. Musa, "Effect of Withania somnifera (Ashwagandha) on the development of chemotherapy-induced fatigue and quality of life in breast cancer patients," Integrative Cancer Therapies, vol. 12, no. 4, pp. 312-322, 2013.

[107] N. Pires, V. Gota, A. Gulia, L. Hingorani, M. Agarwal, and A. Puri, "Safety and pharmacokinetics of withaferin-A in advanced stage high grade osteosarcoma: a phase I trial," Journal of Ayurveda and Integrative Medicine, vol. 11, no. 1, pp. 68-72, 2020. 